\title{
LAS POSIBILIDADES PARA LA EXPERIENCIA DE LA REPRESENTACIÓN ESCÉNICA COMO PRÁCTICA DE OCIO DENTRO DE LA “NUEVA NORMALIDAD” MEXICANA POSPANDÉMICA
}

Recebido em: 10/08/2020

Aprovado em: 15/12/2020

Licença: @) (1) @

Karla Ysolina Uriarte Torres ${ }^{1}$

Universidade Federal de Minas Gerais (UFMG)

Belo Horizonte - MG - Brasil

RESUMÉN: El siguiente texto tiene como objetivo reflexionar sobre la posibilidad de vivenciar la representación escénica previamente concebida para el espacio físico dentro del espacio virtual establecido durante el aislamiento ocasionado por la pandemia del COVID-19 en México, con la finalidad de vislumbrar algunas de sus posibilidades dentro de su Nueva Normalidad. Esto, por medio de un dialogo entre sus características técnicas y tecnológicas, los hábitos establecidos previamente al respecto y la factibilidad del consumo digital dentro de la sociedad mexicana. Observándose como resultado, una dificultad para implementar un espacio exclusivamente virtual para su práctica dentro de la Nueva Normalidad, especialmente, cuando sólo 56.4\% de los mexicanos (as) dispone de acceso a internet.

PALABRAS CLAVE: Representación Escénica. Espacio Virtual. Nueva Normalidad.

\section{THE POSSIBILITIES FOR THE EXPERIENCE OF THE SCENIC REPRESENTATION AS A LEISURE PRACTICE WITHIN THE POST- PANDEMIC MEXICAN "NEW NORMAL"}

ABSTRACT: The following text aims to reflect on the possibility of experiencing the scenic representation, originally conceived for the physical space, within the virtual space established during the isolation caused by the COVID-19 pandemic in Mexico, in order to infer some of its possibilities within Mexican "New Normal. This, through a dialogue between its technical and technological characteristics, the habits previously established in this regard and the feasibility of digital consumption into the Mexican reality. Observing as a result, a difficulty to implement an exclusively virtual space for its practice within the Mexican "New Normal", especially when only $56.4 \%$ of Mexicans have internet access.

KEYWORDS: Scenic Representation. Virtual Space. New Normal.

\footnotetext{
${ }^{1}$ Doutora em Estudos do Lazer pelo Programa de Pós-graduação Interdisciplinar em Estudos do Lazer (PPGIEL - UFMG). Membro do Grupo de pesquisa EDUDANÇA/UFMG.
} 


\section{Introducción}

El presente artículo pretende reflexionar sobre las posibilidades de vivenciar las representaciones escénicas como el teatro y la danza dentro de los espacios virtuales disponibles durante la pandemia, con la finalidad de inferir como sería esta experiencia dentro de la Nueva Normalidad mexicana ante la posible afectación de los espacios físicos reconocidos tradicionalmente.

"Nueva Normalidad" que supone algo diferente por venir, una posible nueva forma de organización del cómo hacer, percibir y juzgar las prácticas y las relaciones sociales, en consecuencia, una posible nueva forma de organizar a los sujetos en el mundo material sea este físico o virtual, a causa del aislamiento social demandado por el COVID-19, que puso, aparentemente en pausa, la forma en que las personas se relacionaban con los otros y su entorno para trabajar, divertirse, cuidar de sí y de los otros significativos.

Es así como, a causa de la expansión del COVID-19 y su aislamiento social voluntario o forzoso, algunos espacios que estaban dispuestos físicamente para la convivencia social de los seres humanos fueron cerrados, entre ellos, aquellos donde se llevaban a cabo las representaciones escénicas, consideradas como tales, aquellas creadas para dialogar con los otros por medio de la apreciación o participación de sus públicos, que permiten generar experiencias consideradas dentro de este trabajo como parte de las prácticas escénicas, entre las cuáles también pueden encontrarse el de la creación, actuación, producción, divulgación y gestión, entre otras opciones, siendo el dialogo posible de esta experiencia, sea de forma activa o interactiva, el punto central de este texto, por ser el más afectada a partir del cierre de sus espacios físicos que limitó las posibilidades de su experiencia a la ofrecida tan solo por los espacios virtuales. 
Considerando como espacios virtuales aquellos realizados fuera del espacio físico que favorecen a la interactividad entre los participantes por medio del establecimiento de diversas redes de comunicación, espacios que a raíz de la pandemia, han sido la plataforma por medio de la cual, las representaciones escénicas que acontecían en físico, han buscado adaptarse y transmutar, acelerando el paso hacia la interactividad que ya había estado desarrollándose dentro de este tipo de entretenimiento desde finales del siglo XX.

De esta forma, se observa cómo hasta antes de la pandemia era posible vivir la experiencia de estas prácticas dentro de los espacios virtuales en paralelo con sus espacios físicos que ahora son inexistentes, ¿será que volverán a reactivarse?, ¿será que nuevos espacios y lugares se generaran a partir de las posibilidades resultantes de la transición de las practicas físicas hacia las virtuales? ¿será que las prácticas fiscas serán desplazadas por las virtuales favoreciendo su extinción?

Con respecto a la diferenciación existente entre los espacios percibidos como nuevos y los viejos, Benedict y Suárez (1993), comentan que, "Lo "nuevo" y lo "viejo" están alienados diacrónicamente, y el primero parece invocar siempre una ambigua venia de los muertos.” (p.260), ante esto, podría contemplarse la posibilidad de que los nuevos espacios pandémicos y pospándemicos para la representación escénica resultantes del aceleramiento virtual, de alguna forma, serían construidos sobre las experiencias históricamente desarrolladas físicamente, por lo tanto, sea esta una ruptura o continuación de lo antes vivenciado, estaría haciendo referencia a lo anteriormente experimentado.

Ahora bien, con respecto al ambiente pandémico mexicano, se ha observado como el COVID-19 ha llegado a permear diferentes dimensiones de la vida humana como la cultural, social, económica, psicológica y biológica, tanto por sus afectaciones a 
la salud como por la nueva distribución de los cuerpos en los espacios y tiempos como consecuencia del aislamiento social voluntario o forzoso, lo que ha ocasionado el surgimiento de nuevos hábitos de higiene y convivencia social que buscan evitar el contacto interpersonal que pueda incrementar el número de contagios por encima de la capacidad de atención médica, situación que, probablemente, ha ido generando nuevas prácticas de vinculación con el entorno material y subjetivo, algunas de las cuáles perduraran dentro de la Nueva Normalidad.

Nueva Normalidad que, al ser aún desconocida, ha ocasionado una incertidumbre económica que ha llevado a la recisión de contratos laborales, despidos o disminución de horas laborales con o sin disminución de salario temporal o permanentemente, así como, a la suspensión de labores educativas de forma presencial y de otros servicios considerados como no esenciales para la sobrevivencia de una nación como aquellos vinculados con el entretenimiento.

En consecuencia, un porcentaje de la población mexicana se ha dispuesto a permanecer en casa y/o salir bajo las restricciones y protección necesaria para evitar contagiarse y/o contagiar a otras personas, aunque, cada vez son menos las que permanecen bajo estas restricciones establecidas de forma obligatoria o voluntaria en la mayoría de los estados de la República, esto debido al paso del tiempo, la inestabilidad del virus y a las decisiones políticas; y con todo a la prohibición de prácticas que promueven la reunión de muchas personas dentro de un mismo espacio físico, como aquellos dispuestos para las representaciones escénicas, importantes por ser manifestaciones culturales que favorecen la convivencia humana de manera lúdica y voluntaria y que tienen el potencial de conducir a transformación personal y por ende social por medio de su representación ante los otros, lo que coloca a esta actividad como una práctica de ocio con base en la definición trabajada por Gomes (2011 apud, 2014). 
Bajo este panorama, si hablar de ocio en México ya es complicado por ser este un país donde parece invisible la existencia de una política pública fuera de la recreación, el deporte o el turismo, aún más, cuando se encuentra acompañado por un confinamiento rodeado por el aire del desempleo y la incertidumbre, que ha conducido, a una aparente urgencia de monetizar cualquier práctica inclusive las de ocio, con la finalidad de tener los recursos económicos para sobrevivir en el presente y en la Nueva Normalidad porvenir.

Posible, gracias a las ventajas que han sido ofrecidas por medio del desarrollo en las últimas décadas de plataformas digitales como Facebook, YouTube, Instagram y recientemente TikTok, a través de las cuales, se ha conseguido democratizar la exhibición de la creatividad y su monetización. Estableciendo durante las últimas décadas una oportunidad de comunicación en red entre sujetos con intereses comunes que, con el paso del tiempo, fueron generando contenido atractivo por su aparente, innovación, originalidad y contemporaneidad que, posteriormente, fue reconocido por el mercado de consumo, es decir, lo que aparentemente comenzó como un espacio de comunicación desmonetizada para quien participaba en ella, fue convirtiéndose en un lugar para la valorización de la exhibición de la creatividad como una vía para la publicidad de productos ya existentes o como un producto en si factible de ser monetizado.

Exhibición de la creatividad anteriormente exclusivas de las practicas escénicas, de las cuáles, fueron surgiendo nuevas formas de ocio como el cine, la televisión, las series, realityshows, los vlogs, entre otras.

Como resultado, se percibe como durante las últimas décadas del siglo XX y las primeras décadas del silgo XXI fueron desarrollándose en paralelo dos espacios con potencialidad diferenciada para ofrecer diversos lugares para la práctica del ocio con 
menor o mayor grado de control percibida sobre la acción, estableciéndose con esto el camino para una ruptura o continuidad entre las practicas físicas y virtuales, proceso que se ha visto acelerado debido al ambiente generado por el COVID-19, dentro del cual, se ha observado el cierre de algunos espacios físicos que han ocasionado la quiebra y posible desaparición de instituciones internacionalmente reconocidas como el Cirque do Solei, al mismo tiempo, que nuevas formas virtuales se han fortalecido, y con ello, nuevas formas de vivenciar las prácticas escénicas a través del uso de las diferentes plataformas tecnológicas disponibles, razón por la cual, el principal interés de este artículo es el de reflexionar sobre las posibilidades existentes para la representación escénica como práctica de ocio dentro del espacio virtual pándemico con el propósito de vislumbrar como podría continuar siendo su experiencia dentro de la Nueva Normalidad mexicana aun ante la sobrevivencia de sus posibilidades ofrecidas dentro del espacio físico.

Para este fin, serán analizados estudios realizados dentro de las artes escénicas que dialoguen con las posibilidades de adaptación y desarrollo virtual de esta práctica en diálogo con los hábitos que con respecto a esta y el espacio virtual, existían de forma previa y durante la pandemia dentro del contexto mexicano.

De esta forma, como preámbulo para esta reflexión, a continuación, será presentado un breve recorrido por el desarrollo técnico y tecnológico que han fundamentado la composición de estas prácticas modernas de ocio, con la intención de identificar sus vínculos con las nuevas formas de creación experimentadas durante el siglo XXI y sus posibilidades para los espacios disponibles en la Nueva Normalidad, seguido de una descripción de los hábitos que con respecto a las prácticas escénicas y virtuales existían en la realidad mexicana previamente a la pandemia, para poder inferir el panorama posible para su vivencia dentro de la Nueva Normalidad. 


\title{
Posibilidades de la Representación Escénica Fuera de su Espacio Físico de Acuerdo con sus Características Técnicas y Tecnológicas
}

De acuerdo con, Guarinos (2008),

\begin{abstract}
El teatro en pleno siglo XXI ya no es sólo una forma de ocio, es un signo de cultura [...] Pero independientemente de la verdad o no de este hecho, lo cierto es que la teatralidad, hoy por hoy, excede al mismo teatro y se expande a otras formas de ocio, otros discursos ficcionales o no y otros medios o manifestaciones artísticas. A la irrupción en nuestras vidas cotidianas de las nuevas tecnologías hay que sumar nuestra tendencia cultural occidental a la hibridación de los últimos tiempos. Y de ese modo también el teatro se ha visto afectado, en el buen sentido de la palabra, por dichas tecnologías y éstas por aquel (p.1).
\end{abstract}

Ante este discurso, se observa como el diálogo entre el teatro, como espacio y práctica, y la tecnología; aparecen como parte de las temáticas a ser tratadas dentro de las prácticas escénicas, en particular, porque los avances tecnológicos han colaborado con la construcción y visibilidad de los espacios y lugares existentes para la representación escénica, que ha llegado hasta el desarrollo de simuladores de salas virtuales que favorecen la apreciación de una obra realizada por avatares o por seres humanos previamente grabados dentro de una escenario existente dentro del terreno físico.

Todo esto factible, gracias a la característica multidisciplinar del evento escénico y a la utilización de recursos multimediáticos para la amplificación de la simulación de lo real y la apariencia (GUARINOS, 2008). Tomando al teatro como referencia de las prácticas escénicas, a razón de ser este, el espacio físico definidor del lugar escogido para su establecimiento como práctica moderna de ocio.

Dialogo anteriormente citado entre el cuerpo expresivo y la tecnología emergente dentro de las prácticas escénicas, que ha permitido potencializar la interacción entre platea y público, instaurando otras posibilidades de vivirla, como, actualmente, es la forma interactiva, la cual, permite una dimensión más diversificada en alcance y de decisión sobre los escenificado, pasando así de una interacción bilateral 
establecida entre el público y el creador escénico, para una interactividad donde la posibilidad de establecer redes continuas de participación se expande hacia diversos públicos y actores situados en diferentes espacios tanto físicos como virtuales, llegando incluso, a diluirse cada vez más la línea entre actor y público, particularmente, cuando este último tiene la posibilidad de tomar parte dentro de la creación escénica. Observándose como el dialogo entre tecnología y la escena siempre ha existido, de igual forma que la necesidad constante de transformar los modos de producción y representación escénica para la consecución de una comunicación más cercana con el público asiduo y deseado.

De este modo, para comprender como estas posibles relaciones entre la experiencia escénica y las nuevas tecnologías han estado siempre existentes entre las prácticas escénicas, es importante recordar cómo este dialogo partió de un espacio físico proyectado para un mundo moderno en constante progreso. Donde la escena era construida a partir de la experiencia generada por los ambientes y acciones producidos un escenario, sin embargo, con independencia a su estructura física. Con base en esto, el espacio escénico podría ser concebido más allá del espacio físico teatral y sus prácticas.

Separación de la escena de su espacio físico, principalmente, sustentada por la transición hacia un pensamiento posmoderno, donde se observa, un alejamiento del uso exclusivo de la escena por el espacio teatral, que había sido predominantemente italiano en Latinoamérica del siglo XIX y de la primera mitad del siglo XX, por ser este, una estructura arquitectónica que permitiría una mejor visibilidad, acústica, transformaciones escénicas y efectos de ilusiones (ROUBINE; MICHALSKY, 1998). Siendo, actualmente, concebido y percibido de manera diferente gracias a las transformaciones presentadas por los modos de producción y consumo a lo largo del siglo $\mathrm{XX}$ que, favorecieron la construcción de obras de escénicas con base en una 
libertad creativa favorecida por la posibilidad de ocupar nuevas formas, tiempos y espacios, y mayor conciencia de su entorno social y político (MALAGÓN, 2012).

Con lo anterior, se percibe, como la práctica escénica ha sido concebida desde su inicio para ser observada por otros, identificados como público, a través, de un espacio reconocido como escena que puede llegar a sobrepasar el espacio construido físicamente a partir de las identidades construidas por medio de la representación y a partir del aprovechamiento de los avances científicos y tecnológicos que han ido desarrollando formas para mostrar el acontecimiento escénico, el cual puede llegar a ser espectacular por su capacidad de simular o aparentar la realidad dentro de un espacio paralelo al percibido como real, o por la creación de ambientes surreales percibidos como fuera de este mundo (WILLIAMS, 2011).

Materialización de realidades lejanas o cercanas en espacio y/o tiempo, que ha llevado a percibir como espectáculos a estas representaciones escénicas, debido a que, "La realidad objetiva está presente en ambos lados. Cada noción así fijada no tiene otro fondo que su paso a lo opuesto: la realidad surge en el espectáculo y el espectáculo es real." (p.26), espectáculo que busca subsistir de forma independiente de su creador por tiempo indefinido, sin embargo, sujeto a las condiciones y fines del sistema reinante y sus prácticas (DEBORD, 2007). Espectáculo que, al ser llevado a su máximo esplendor para hacerlo más visible por medio de los recursos tecnológicos, mayor podría ser la espectacularidad que lo dirija hacia el distanciamiento de su propia realidad sustentadora y razón de origen (WILLIAMS, 2011).

Ante esto, la factibilidad de ser consumida esta práctica por su espectacularidad crece cuando el producto está más cercano al uso excesivo de los recursos tecnológicos y más alejado del contexto real y del contenido que le dio forma, y "cuanto más superficial es la percepción, menor es el placer que se obtiene del espectáculo 
contemplado, más atrae la búsqueda de lo "sensacional", el culto de la hazaña obvia y de la virtuosidad visible [...]"( BOURDIEU, 1993, p.69). Bajo esta perspectiva, las posibilidades de ser atraído o repelido por la apariencia y simulación de una composición escénica estarían articuladas a la proximidad y nivel de conocimiento poseído con respecto a cada uno de los componentes que la conforman, es decir, iría a depender de si la educación sensible para la experiencia escénica se encuentra fundamentada en un menor o mayor grado en los avances tecnológicos que colaboraron para su visibilidad o en el argumento, composición y forma de representar de la obra.

De esta manera, se puede observar como la representación escénica y las nuevas tecnologías han caminado de la mano para la optimización de la visibilidad de un evento creado a partir de la realidad percibida y, por lo tanto, factible de ser espectacular conforme más se asemeja o distancia de esta. Cambios que han ocasionado ciertas mutaciones en las formas en que las prácticas escénicas son experimentadas, aparentemente, sin perder su carácter multidisciplinar y multimediático.

Por lo tanto, con base en esto, se puede inferir que, la representación escénica, precisamente por su carácter multidisciplinario y multimediático nato, pueden transmutar hacia otros espacios físico o virtuales sin necesitar de la presencia física de un escenario en específico, sobre todo, cuando se han presentados algunos avances en las posibilidades virtuales para su experiencia previamente a la pandemia que podrían facilitar su trasposición o transmutación para este espacio, como consecuencia, del desaparecimiento momentáneo de las formas y modos físicos disponibles para la representación escénica que ha llevado a plantearse la posibilidad de su extinción y, también, acelerado la motivación para la construcción de nuevas formas de crear dramaturgias para la escena virtual y con ellas nuevas formas de vivirlas dentro de ambientes hipermediáticos y transmediáticos, que, posiblemente, continuaran siendo 
perfeccionados para crear condiciones similares a aquellas ofrecidas de forma física. Siendo entendida como experiencia escénica hipermediática, aquella que se ofrece a partir de una dramaturgia que construye posibilidades para que el público pueda elegir sus propias opciones de lectura y experiencia escénica a partir de la elaboración de un hiper texto, y como transmediática, aquella que se construye por medio de diferentes plataformas virtuales de comunicación, a través de las cuáles, se busca experimentar el diferente contenido existente sobre una misma dramaturgia, de manera independiente y/o complementaria (ROSALES; MONTES, 2016). Ambas, opciones dirigidas hacia el fomento de una interactividad entre creadores y público que rebasa las posibilidades de interacción preestablecidas dentro del espacio físico.

Sin embargo, ¿será la misma experiencia escénica la que se vive dentro de un espacio físico y la que se vive mediante el espacio virtual? ¿Cuáles son las posibilidades de que su presencia física desaparezca, al menos, de la forma en como era vivida antes de la pandemia?, Posiblemente, esto dependerá de la apropiación desarrollada alrededor de estas prácticas previo al aislamiento social y a la apropiación que de las plataformas virtuales generadoras de los nuevos posibles espacios virtuales se tenga. Por tal motivo, a continuación, serán descritas las posibilidades de su arraigo de forma física y de acceso a las plataformas virtuales dentro de la sociedad mexicana.

\section{Panorama de la Representación Escénica como Práctica de Ocio en México del}

\section{Siglo XXI}

Tomando como punto de partida las prácticas escénicas modernas, estas se fueron estableciendo en México bajo una mezcla del modelo europeo y las reminiscencias de las costumbres precoloniales desde el siglo XIX, estando entre las prácticas más reconocidas hegemónicamente la danza bajo la forma del ballet, el teatro 
y la ópera, y entre las prácticas populares el teatro de carpa y revista. Siendo ambos discursos escénicos construidos sobre la base del imaginario romántico que buscaría colaborar con el establecimiento de una identidad nacional a partir de la educación sensible de forma más o menos crítica, principalmente, cuando peligraba la delimitación territorial del país tanto por el norte como por el occidente.

Sensibilidades que buscarían abarcar los espacios destinados a la educación por medio de la formación artística y las prácticas escénicas representativas de ese México imaginado entre su pasado histórico y su futuro moderno. Prácticas que perfumadas por el espíritu posrevolucionario consiguieron colaborar con el establecimiento de lo que actualmente es percibido por los propios y ajenos como mexicano, aparentemente, perpetuado hasta nuestros días de forma oficial en vez de seguirse reinventado conforme lo demandan las nuevas identidades que han ido surgiendo a raíz de la reivindicación étnica y del mundo globalizado.

De este modo, con la llegada del nuevo sexenio gubernamental en 2018, la política destinada a las prácticas escénicas ha sido receptora de recortes presupuestales para nuevas creaciones y de amenazas de una posible restructuración institucional, mientras que, al mismo tiempo, pareciera que se intenta incrementar el turismo en nuestro país a través de la venta de una cultura que busca representar a un México a partir de los vestigios de las prácticas escénicas de un pasado nacionalista.

Sin embargo, ya que, el tema de este artículo se focaliza en las representaciones escénicas como la danza y teatro, y sus posibilidades de práctica durante los momentos de ocio disponibles como consecuencia del aislamiento social provocado por el COVID-19, más allá de comprobar el valor reconocido de la cultura por este y otros gobiernos anteriores, a continuación, serán utilizadas estadísticas oficiales con respecto a la producción y consumo cultural, con la intención, de crear un ambiente contextual 
mexicano contemporáneo que ayude a comprender como ha sido la continuidad o discontinuidad de estas prácticas escénicas dentro de los espacios pre y pándemicos. Para tal propósito, se tomaron como referencias diversas estadísticas oficiales proporcionadas por los sitios en línea del Instituto Nacional de Estadística, Geografía e Informática y por la Secretaría de la Cultura, con plena conciencia de su parcialidad para la representación de una realidad completa de las prácticas escénicas mexicanas, no obstante, importantes por ser a partir de estas que se va construyendo la política cultural que las promueve y visibiliza.

De esta forma, primeramente, se observa cómo para la Cuenta Satélite de la Cultura de México (CSCM) 2108 (INEGI, 2019a), las prácticas escénicas se encuentran localizadas dentro de las actividades culturales, específicamente, dentro de la categoría correspondiente a las artes escénicas y espectáculos, que contempla a

[...] los eventos y espectáculos culturales en vivo relacionados con el teatro, la danza, la ópera, espectáculos artísticos y culturales en general (incluidos los deportivos), además de algunos servicios como los prestados por promotores y agentes, y el alquiler de espacios para presentar los eventos (INEGI, 2019a, p.12).

Prácticas de artes escénicas que concebidas conjuntamente con otro tipo de espectáculos deportivos, ocupan un lugar intermedio dentro del PIB nacional que, en 2018 fue del $2,1 \%{ }^{2}$ aproximadamente, aportando con esto, el 5.5\% sobre el $100 \%$ obtenido por la producción cultural en general, siendo los medios audiovisuales los que ocuparían el primer lugar con una aportación del 36.8\%, estando entre estas actividades, las realizadas por los medios de difusión e industrias de cine, radio, televisión, filmes, videojuegos, prácticas ofertadas por internet; y por las videotecas, cinetecas y prestación de servicios de autor, de distribución y regulación de los productos culturales, en cuanto que, la música y los conciertos se localizarían en el último lugar, con una aportación del $1.1 \%$ (INEGI, 2019a).

\footnotetext{
${ }^{2}$ De acuerdo con la página web: https://datosmacro.expansion.com/pib/mexico
} 
En cuanto al gasto realizado para el desarrollo de la oferta cultural, se observó que en las artes escénicas este fue de un $5.0 \%$, mientras que para los medios audiovisuales fue de $38.1 \%$ y, para la música, conciertos, artes visuales y plásticas de $1.5 \%$ (INEGI, 2019a).

Valor y gasto en los bienes y servicios culturales que podrían corresponder a un interés manifestado por su oferta y demanda, que en su conjunto y motivado por el consumo privado alcanzaron un incremento del 3.5\% de 2017 para 2018, obteniendo un valor de 1 millón 289 mil 800 millones de pesos, los cuales fueron derivados del 95.1\% de la producción nacional, caracterizada por ser consumida en un $30.2 \%$ de forma intermedia a través de la adquisición de bienes y servicios para la creación de nuevos bienes y servicios, así como, por un $66 \%$ de consumo como producto final provenientes de los hogares y el gobierno, de un $2.3 \%$ como inversión y de un $1.4 \%$ como exportación (INEGI, 2019a).

Hábitos, prácticas y consumos culturales que sustentarían el valor y gasto de las prácticas culturales en general y de las escénicas en particular, pudiendo caber estas últimas con mayor intensidad dentro de la demanda intermedia y producto final por su experiencia efímera y abierta. Observándose como durante ese periodo existió una mayor inversión en las prácticas audiovisuales, las cuáles, presentarían la mayor aportación al PIB nacional como producto cultural.

Asimismo, con la intención de identificar cuáles son las prácticas de ocio relacionadas con las prácticas escénicas que podrían estar sustentando su baja aportación al PIB nacional, se buscó información dentro de la página web de la Secretaria de Cultura, donde el único estudio disponible correspondería a una encuesta realizada a nivel nacional en 2010 por el Consejo Nacional para la Cultura y las Artes (CONACULTA, 2010), a partir del cual, fue sustraída la siguiente información que 
podría servir de guía para conocer la posible preferencia de la población mexicana por el teatro y la danza como prácticas escénicas de ocio.

De esta forma, se observó cómo el 32.7\% de la población mexicana manifestó asistir a presentaciones de danza siendo el $70 \%$ de forma gratuita, mientras que del $31.3 \%$ que asistiría a ver la oferta teatral, el $28.9 \%$ lo realizaría gratuitamente, de igual modo, dentro de la misma encuesta se observó como el cine considerado como un medio audiovisual para el entretenimiento, fue el que presentó mayor porcentaje de práctica con un $75.2 \%$. Asimismo, un $12.5 \%$ de la población expresaría haber asistido a centros culturales al menos una vez en su vida y un $43.1 \%$ a festividades tradicionales, lugares donde, en ocasiones, serían realizadas algunas de las prácticas escénicas de interés para este artículo (CONACULTA, 2010).

Ahora bien, dentro de los intereses educativos que podrían estar conformando un público y creadores para la oferta y demanda escénica mencionada, un 13.0\% manifestó que le gustaría estudiar danza, un 2.2.\% había estudiado danza y un 3.8\% había practicado danza tradicional, mientras un $7.1 \%$ mostró interés por el teatro y un $1.3 \%$ manifestó alguna vez haber estudiado teatro, siendo la música con un $20.7 \%$ la actividad que mayor interés mostró dentro de esta encuesta, manifestando el $15.9 \%$ de los encuestados saber tocar un instrumento y un $1.5 \%$ haber estudiado alguna vez música (CONACULTA, 2010).

Con base en esto, puede observarse como durante el 2010 fue el cine una de las prácticas realizadas con mayor intensidad, sin tomar en consideración su práctica por medio de las plataformas existentes en línea, ni otras prácticas audiovisuales similares ofertadas de manera virtual que, para aquella época ya existían, y que, tal vez, al ser consideradas, se hubiera podido observar su presencia dentro del gusto del público a una década de diferencia con el pándemico 2020. Asimismo, se observa como a 
diferencia del consumo del cine que se caracteriza por ser privado, las prácticas escénicas como la danza y el teatro, al menos dentro de sus espacios físicos, fue consumida con mayor intensidad de forma gratuita, lo que podría estar relacionado con su menor aportación al PIB como producto cultural.

Por otro lado, el Instituto Nacional de Estadística, Geografía e Informática (INEGI), realiza por medio del Módulo sobre eventos culturales seleccionados, un estudio en 2019 para conocer la participación cultural de la población en el país durante los últimos 12 meses previos al 20 de mayo de 2019, brindando información a las instancias gubernamentales y público general para el desarrollo de la política cultural del país. Para este fin, fue considerada la asistencia a eventos culturales fuera del hogar bajo los términos de asistencia-recepción estipulados por la UNESCO en el manual Como medir la participación cultural, siendo estos los siguientes: la asistencia a obras de teatro, conciertos o presentación de música en vivo, así como a espectáculos de danza, exposiciones y proyección de películas o cine.

Como resultado de este levantamiento de datos realizado en 2336 viviendas del agregado urbano en 32 áreas del país, se observó cómo el 57.8\% de personas mayores de 18 asistió a algún evento cultural, siendo el 59.3\% hombres y el 56.4\% mujeres. De los cuáles, el 24.9\% no contaba con estudios de educación básica, el 59.0\% contaba con educación básica y por lo menos con un año de educación media y el 79.2\% contaba al menos con un grado de educación superior. Encontrándose entre las actividades más practicadas el cine con un $88.3 \%$, seguido de la música con un $47.0 \%$, las exposiciones con un $26.2 \%$, la danza con un $22.5 \%$ y por último el teatro con un $21.4 \%$. Siendo el cine la actividad más atractiva con un $62.9 \%$ y las menos atractivas el teatro con un $73.8 \%$ y la danza con un $75.6 \%$. (INEGI, 2019c) 
De igual modo, se percibió como las redes sociales y el internet eran el medio de difusión con mayor fuerza para dar a conocer las actividades culturales, así como la educación en casa y la escuela aparecieron como factores de estímulo importantes.

De esta forma, se puede observar como la oferta de teatro y danza para los momentos de ocio, principalmente, aquella destinada para ser apreciada dentro de espacios y lugares físicos, ha ocupado un lugar de medio a bajo dentro de las preferencias de las personas que viven dentro del agregado urbano durante las primeras décadas del siglo XXI. Esto, con todo al predominio de un nivel superior de estudios entre los participantes más activos culturalmente, lo que tal vez indicaría, la continuación de una predominancia de la educación audiovisual por encima de la educación existente para las prácticas escénicas de forma física.

Ante esta aparente disminución en el interés por las prácticas escénicas y aumento de las prácticas audiovisuales, ¿hasta dónde será factible la búsqueda por este tipo de actividades escénicas dentro de los nuevos espacios y lugares virtuales disponibles durante y posterior a la pandemia?

\section{La Representación Escénica y sus Posibilidades dentro del Espacio Virtual, ¿Una} Ventaja para Resituarla dentro de las Prácticas de Ocio Mexicanas dentro de la Nueva Normalidad?

Como se ha visto anteriormente, previamente a la pandemia la búsqueda por la experiencia escénica a través de sus representaciones físicas estaba a la baja en comparación con otras prácticas audiovisuales, las cuales, han proporcionado mayores ganancias como producto cultural a nivel nacional. Por lo tanto, se podría pensar que, ante la posibilidad de una ampliación en la presencia de las prácticas escénicas dentro de los medios audiovisuales a través de sus formatos virtuales, podría presentarse un 
incremento de su consumo, no obstante, para que esto ocurriese, también tendría que ser considerado las posibilidades que, a nivel nacional, existen para acceder a este tipo de plataformas localizadas a través de internet.

No sin antes, tomar en cuenta la existencia de las representaciones escénicas también dentro de otros medios audiovisuales más tradicionales como la televisión, mediante la cual, se han reproducido obras de teatro y danza durante la pandemia. Ante esto pareciera que se tendrían dos opciones para el disfrute en casa de las representaciones escénicas de forma digital, aquella ofertada por la televisión abierta y privada y aquella facilitada por las plataformas virtuales localizadas dentro del ciberespacio. Por lo tanto, se verá a continuación las posibilidades de acceso a cada una de estas.

De acuerdo con el INEGI (2019b) en 2019 92.5\% de la población mexicana contaba con televisión, de los cuáles, $45.9 \%$ tendría acceso a servicios de paga; en contraste, $44.3 \%$ de la población contaba con computadora en el hogar, $56.4 \%$ con conexión a internet y un $75.1 \%$ era usuario del teléfono celular. A partir de lo cual, se puede observar como la vía de mayor acceso al espacio digital para el disfrute de las representaciones escénicas sería la televisión, siendo tal la importancia de este medio dentro de la sociedad mexicana que ha llevado a su elección como principal medio de comunicación por la Secretaria de Educación Pública para la impartición de las clases durante el primer semestre del ciclo escolar 2020-2021, junto con la radio para las clases en lenguas indígenas originarias.

Ahora bien, con respecto a internet, de acuerdo con el comunicado de prensa realizado el 14 de mayo de 2020 por el INEGI, del 56.4\% que dispone de conexión de internet en sus hogares, el $70.1 \%$ se declara como usuario entre los seis y más años de edad, siendo el teléfono celular la forma más factible de mantener contacto con el 
ciberespacio debido a que es la tecnología con mayor penetración nacional con un total aproximado de $75.1 \%$ de usuarios, de los cuáles, el $88.1 \%$ posee celular inteligente, lo que les permitiría conectarse a internet en un $90.6 \%$ a través de datos móviles y en un 9.4\% sólo por WI-FI. Estando entre las principales actividades realizadas en 2019 por medio de las plataformas dispuestas en internet, aquellas dirigidas hacia el entretenimiento en un $91.5 \%$, a la información en un $90.7 \%$ y a la comunicación en un 90.6\%. Predominando su práctica dentro del área urbana donde existe un $76.6 \%$ de usuarios virtuales contra el $47.7 \%$ existente en el área rural.

En específico, de acuerdo con We are social ${ }^{3}$ 2020, durante el 2019 el tiempo invertido por el mexicano (a) en internet fue de 8 horas y 21 minutos en promedio, siendo el mayor número de horas utilizado en medios sociales y para asistir contenido de TV en línea. Estando entre le contenido más consumido los videos en un $99 \%$, la música en streaming en un $72 \%$, la radio digital en un $49 \%$ y podcasts en un $46 \%$. Siendo las plataformas más utilizadas: en un 96\% YouTube, 94\% Facebook, 89\% Whatsapp, 78\% FB Messenger, 71\% Instagram, 61\% Twitter, 46\% Pinterest, 36\% Linkedin, 35\% Snapchat, 32\% Skype, 22\% Tumblr, 22\% Twitch, 21\% WeChat, 20\% TikTok, $17 \%$ Reddit y $16 \%$ Line.

Comportamiento digital que a partir de la pandemia se ha incrementado en México de acuerdo con la compañía de investigación de marketing en internet Comscore, particularmente, bajo intereses de búsqueda inclinados hacia la información, educación, videojuegos, entretenimiento y salud, (TECHBIT, 2020), de igual forma que el e-commerce a crecido, debido al cierre o restricciones para el tránsito dentro de lugares públicos destinados al comercio como restaurantes, supermercados y tiendas departamentales, entre otros.

\footnotetext{
${ }^{3}$ Con base en el resumen realizado por esta empresa concebida como una agencia creativa liderada socialmente para conectar personas y marcas de manera significativa.
} 
Ante este panorama, se puede observar como las posibilidades para vivenciar las representaciones escénicas durante la pandemia rondan alrededor del espacio digital establecido por las posibilidades de reproducción brindadas por la televisión pública y de paga, así como por el espacio virtual generado en las plataformas disponibles en línea, las cuales, ponen a disposición tanto la reproducción de espectáculos representados en vivo sea de forma pregrabada o en vivo y de forma gratuita; generando experiencias que, como hemos visto, ofrecen la posibilidad de transgredir el lugar ocupado tradicionalmente como público de forma física, esto, por medio de la creación de espacios y lugares que favorecen la interactividad escénica donde se puede elegir el cómo, cuando, donde y cuantas veces se lleva a cabo la acción representativa, haciendo del producto final una eterna creación inacabada en constante edición.

Sin embargo, como hemos visto anteriormente, está última no sería la posibilidad más factible para vivir la experiencia de las representaciones escénicas ni durante ni después de la pandemia, debido a que existe todavía un rezago en la democratización del acceso a internet que, posiblemente, facilitaría la demanda aún de los espacios físicos como opción para su experiencia dentro de los momentos de ocio dispuestos para la Nueva Normalidad mexicana. Esto sin contar, las dimensiones psicológicas que podrían estar involucradas en esta demanda, como la urgencia de salir y ocupar el espacio público físico y de vivir prácticas que colaboren con la sensación de estar acompañado en el terreno físico.

En consecuencia, la transmutación para los espacios es factible cuando se observa que dentro de los hábitos digitales predomina el uso de Youtube o Facebook plataformas amigables con las formas de hacer, percibir y juzgar practicadas antes de la pandemia, las cuáles incluirían la construcción de un ambiente escénico por medio de la plástica y una narrativa dramática sea a través de la palabra o en movimiento factible de 
ser transmutable al formato de video e imagen como ya ha ocurrido anteriormente cuando ante la invención de la cámara de filmación y la televisión. Transmutación hacia los espacios virtuales de la representación escénica en sus formas de danza, teatro e hibridaciones que podría favorecer la ampliación de su práctica tanto como público activo o interactivo al ver expandidas sus posibilidades dentro del mercado de consumo.

No obstante, la realidad es que existe todavía una limitación de acceso a estos espacios que dificultarían la completa desaparición de los espacios físicos disponibles para su práctica, dentro de los cuáles, se debería seguir fomentando su práctica de forma tradicional a la par de nuevas posibilidades hibridas resultantes de su dialogo con la tecnología y de las experiencias adquiridas durante la pandemia. Especialmente, por ser esta una práctica de ocio que históricamente ha colaborado para la construcción, deconstrucción, divulgación y reivindicación de las identidades mexicanas.

\section{Algunas Consideraciones Finales}

A lo largo de este texto, se buscó reflexionar sobre las posibilidades de vivir la experiencia de la representación escénica como práctica de ocio durante la pandemia y dentro de la futura Nueva Normalidad, como consecuencia del escenario construido por el desaparecimiento momentáneo de los espacios físicos tradicionales a causa del aislamiento social voluntario o forzoso como medida de prevención ante la amenaza del COVID-19.

Es así como, a partir de una reflexión donde se observó la factibilidad de llevar la representación escénica física para el espacio virtual, se observó que técnica y tecnológicamente sería posible esta transición, esto sin considerar la calidad y los modos de consumo. Con la posibilidad de transmutar hacia otros nombres, ya que, basta 
recordar como de la hibridación del teatro con los avances tecnológicos han ido surgiendo nuevas prácticas como el cine.

Sin embargo, esto dista de amenazar la desaparición de su forma física, principalmente, cuando más del $40 \%$ de la población aún vive desconectado de la realidad virtual, aunque, su forma física de consumo si podría verse alterada a causa de las nuevas normas de higiene y distanciamiento social provenientes del COVID-19, principalmente, porque ante la disminución del aforo no sería redituable para algunos productores la reactivación de los espacios físicos y su oferta, siendo el aumento de los costos de entrada también poco factible en plena recesión económica.

Por consiguiente, se prevé una inclinación por continuar desarrollando nuevas formas virtuales de representación escénica por ser estas redituables para quien las produce, debido a que, como se ha visto al inicio de este texto, es posible monetizar la exhibición de la creatividad propia de las prácticas escénicas dentro del espacio virtual, además de existir la posibilidad de cobrar en línea para su consumo en directo, lo que irá delineando las bases para una nueva educación sensible para estas prácticas y sus modos de producción y consumo, formando una demanda dirigida en mayor grado hacia la búsqueda de un producto escénico interactivo, aspecto a considerar en dado caso se desee reactivar y fortalecer el consumo de las representaciones escénicas a través de sus espacios físicos para su experiencia dentro de la Nueva Normalidad mexicana.

\section{REFERENCIAS}

BENEDICT, A; SUÁREZ, E.L. (Traducc.). Comunidades Imaginadas. Reflexiones sobre el origen y la difusión del nacionalismo. México D.F.: Fondo de Cultura Económica, 1993. 315 p.

BOURDIEU, P. Deporte y clase social. In: BROHM, J.M.; BOURDIEU, P. Materiales de sociología del deporte. Madrid: Ediciones la piqueta, 1993, 188 p.

CONACULTA. Encuesta Nacional de hábitos, prácticas y consumo culturales. 
Comparativo estatal de resultados básicos. México: CONACULTA, 2010. Disponible en: http://www.cultura.gob.mx/encuesta_nacional/\#.XzHkXyhKjIU. Acceso en: 30 jun. 2020.

DEBORD, G. La sociedad del espectáculo. 2. ed. Rosario: Kolectivo Editorial "Ultimo Recurso", 2007. 140 p.

GOMES, C. L. Lazer: necessidade humana e dimensão da cultura. Revista Brasileira de Estudos do Lazer. Belo Horizonte, v.1, n.1, p.3-20, jan./abr. 2014. Disponible en: https://periodicos.ufmg.br/index.php/rbel/article/view/430 . Acceso en: 04 jul. 2020.

\begin{tabular}{llllll} 
& Estudos do Lazer e geopolítica do conhecimento. Revista Licere. \\
\hline Belo & Horizonte, v.14, n.3, p.1-25, set. & 2011. Disponible en:
\end{tabular} http://www.anima.eefd.ufrj.br/licere/sumario.html?ed=29.

GUARINOS, V. Teatro, Comunicación y Nuevas Tecnologías. ICONO 14, n. 18, p.1-7, 2008. Disponible en: http://www.redalyc.org/articulo.oa?id=552556592001. Acceso en: 26 jun. 2020.

INEGI. Cuenta Satélite de la Cultura en México, 2018. México: Comunicado de prensa INEGI, 2019a, 13 p. Disponible en: http://www.inegi.org.mx/contenidos/saladeprensa/boletines/2019/StmaCntaNal/CSCltur a2019.pdf. Acceso en: 30 jun. 2020.

- Encuesta Nacional sobre Disponibilidad y Uso de Tecnologías de la Información en los Hogares (ENDUTIH) 2019. México: INEGI, 2019b. Disponible en: http://www.inegi.org.mx/programas/dutih/2019/. Acceso en: 02 ago. 2020.

$\begin{array}{cccc} & \text { Modulo sobre eventos culturales selecionados. Nota Técnica. México: } \\ \text { INEGI, } & \text { 2019c. } & \text { Disponible } & \text { en: }\end{array}$ http://www.inegi.org.mx/contenidos/programas/modecult/doc/nota_tecnica_modecult_ may2019.pdf. Acceso en: 2 jul. 2020.

Estadísticas a propósito del día mundial del internet (17 de mayo) datos nacionales. México: Comunicado de prensa INEGI, 2020. 7 p. Disponible en: http://www.inegi.org.mx/contenidos/saladeprensa/aproposito/2020/EAP_Internet20.pdf. Acceso en: 08 ago. 2020.

MALAGÓN, R. De la tradición clasicista a la experimentación moderna: la historicidad de la noción de creatividad. Logos, Revista de la Facultad de Filosofía y Humanidades, Bogotá, Colombia, n. 21, p.11-44, ene.-jun., 2012. Disponible en: https://studylib.es/doc/7877130/de-la-tradici\%C3\%B3n-clasicista-a-laexperimentaci\%C3\%B3n-moderna--la. Acceso en: 20 jul. 2020.

ROSAlES, M. A. G.; MONTES, M. J. S. Posibilidades de un teatro transmedia. Artnodes, Revista de arte, ciencia y tecnología, n. 18, 2016, p.64-72, 2016. Disponible en: http://artnodes.uoc.edu/. Acceso en: 04 jun. 2020.

ROUBINE, J. J.; MICHALSKI, Y. A linguagem da encenação teatral. 2. ed. Rio de Janeiro: J. Zahar, 1998. 237 p.

TECHBIT. ¿Cómo es el consumo digital em México en el marco de la pandemia? El 
Universal. México, 05 may. 2020. Disponible en: http://www.eluniversal.com.mx/techbit/como-es-el-consumo-digital-en-mexico-en-elmarco-de-la-pandemia. Acceso en: 02 ago. 2020.

UNESCO. Como medir la participación cultural. Montreal, Canadá: UNESCO, 2014. 132 p. Disponible en: https://observatoriocultural.udgvirtual.udg.mx/repositorio/bitstream/handle/123456789/ 371/ComoMedirParticipacionCul_unesco.pdf?sequence=1\&isAllowed=y. Acceso en: 2 jul. 2020.

WE ARE SOCIAL. Digital 2020 México. Disponible en: http://datareportal.com/reports/digital-2020-mexico. Acceso en: 27 jun. 2020.

WILLIAMS, R. Cultura e materialismo. São Paulo: UNESP, 2011. 380 p.

\section{Endereço da Autora:}

Karla Ysolina Uriarte Torres

Calle 55 \#695-F por 88 y 90

Fraccionamiento Los Almendros Ciudad Caucel, Mérida

Yucatán - México

Endereço eletrônico: isotorres04@gmail.com 